University of Nebraska - Lincoln

DigitalCommons@University of Nebraska - Lincoln

Faculty Publications, Department of Psychology

Psychology, Department of

March 2001

\title{
Environmental Familiarization in Rats: Differential Effects of Acute and Chronic Nicotine
}

Rick A. Bevins

University of Nebraska-Lincoln, rbevins1@unl.edu

Jana Koznarova

University of Nebraska-Lincoln

Tracy J. Armiger

University of Nebraska-Lincoln

Follow this and additional works at: https://digitalcommons.unl.edu/psychfacpub

Part of the Psychiatry and Psychology Commons

Bevins, Rick A.; Koznarova, Jana; and Armiger, Tracy J., "Environmental Familiarization in Rats: Differential Effects of Acute and Chronic Nicotine" (2001). Faculty Publications, Department of Psychology. 149.

https://digitalcommons.unl.edu/psychfacpub/149

This Article is brought to you for free and open access by the Psychology, Department of at DigitalCommons@University of Nebraska - Lincoln. It has been accepted for inclusion in Faculty Publications, Department of Psychology by an authorized administrator of DigitalCommons@University of Nebraska - Lincoln. 


\title{
Environmental Familiarization in Rats: Differential Effects of Acute and Chronic Nicotine
}

\author{
Rick A. Bevins, Jana Koznarova, and Tracy J. Armiger \\ Department of Psychology, University of Nebraska-Lincoln, Lincoln, Nebraska 68588-0308
}

\begin{abstract}
If an environment is familiar, rats will interact more with a novel object than if the environment is unfamiliar. In two experiments we used this behavioral tendency to assess the effects of nicotine on environmental familiarization (i.e., an elevated platform). As expected, rats given 2 min of exposure to the platform on 2 consecutive days (familiarization phase) interacted more with a novel object in a subsequent test than rats that had not experienced the platform until the test day. During the familiarization phase acute pretreatment with nicotine $(0.6$ and $1.8 \mathrm{mg} / \mathrm{kg}$, subcutaneous $)$ 10 min before platform exposure interfered with familiarization processes, as measured by object interaction on the drug-free test day. Behavioral measures of activity (e.g., turning and midline crosses) eliminated an account based on nicotine-induced motor impairment. Furthermore, this effect of acute nicotine on familiarization was not due to nonspecific effects of nicotine. Controls that received equivalent nicotine exposure temporally separated from platform exposure interacted more with the novel object than similarly treated controls that were unfamiliar with the platform on the test day. Interestingly, rats treated once daily with $0.6 \mathrm{mg} / \mathrm{kg}$ nicotine for 14 days before the familiarization phase (chronic condition) did not show a decrease in environmental familiarity. This dissociation extends a growing literature finding that the behavioral and neurobiological effects of nicotine differ, in part, after acute and chronic exposure. Indeed, acute nicotine $(0.2,0.6$, and $1.2 \mathrm{mg} / \mathrm{kg})$ in the present report consistently decreased the amount of time spent with one paw on the edge of the platform; chronic nicotine did not affect this behavior.
\end{abstract}

Key Words: acetylcholine; activity; cholinergic; habituation; learning; memory; nicotinic.

Funds from the National Institute of Health (DA 11893), the Nebraska Cancer and Smoking Disease Research Program, and the University of Nebraska-Lincoln Research Council supported the research in the present report. TJ.A. was supported by a Howard Hughes Medical Research Grant while conducting some of this research. We thank Katherine Pickett and Grey Pickerill for their technical assistance and Eliot Hearst for donating the white noise generator. We are also grateful to Joyce Besheer, Daniel Leger, and Matthew Palmatier for their thoughtful comments on an earlier version of this report.

Address correspondence and reprint requests to Rick A. Bevins, Department of Psychology, University of Nebraska-Lincoln, Lincoln, NE 68588-0308. Fax: (402) 472-4637. E-mail: rbevins1@unl.edu. 


\section{INTRODUCTION}

Much of the neurobiological research on learning and memory has used animal models that involve explicitly programmed contingencies between stimuli (Pavlovian conditioning) or between behavior and stimuli (operant conditioning). Examples include eyeblink conditioning (Gould \& Steinmetz, 1996), fear conditioning (LeDoux, 1998), the radial arm maze (Levin et al., 1996), the delayed matching-to-sample task (Buccafusco et al., 1995), and drug self-administration (Goeders \& Guerin, 1996). Interestingly, embedded within each of these preparations is a learning phenomenon that has not received as much empirical attention-environmental familiarization. Environmental familiarization is often evidenced by changes in behavior with repeated experiences with an environment. These behavioral changes presumably require neural storage of the stimulus elements/features that compose the environment [see Kimble (1961) for a detailed discussion of the definition of learning].

Numerous laboratories have shown that rats display a decrease in general activity with continued exposure to an environment (Aloisi et al., 1997; Bindra \& Spinner, 1958; Glickman \& Hartz, 1964; Mason et al., 1998; Montgomery, 1953). In a classic study, Montgomery (1953) found that rats in an $\mathrm{H}$-shaped maze decreased the number of quadrants visited with repeated exposure to the maze. This decrease in activity was thought to reflect the degree of environmental familiarity. Interestingly, when one feature of the $\mathrm{H}$ maze was systematically manipulated (i.e., shade of maze walls), the number of quadrants visited increased as the difference between the shade of the familiar maze (e.g., black) differed from a similarly constructed testing maze (e.g., white or gray).

Behavioral changes induced by an unfamiliar environment in rats have clear neurochemical correlates. The GABAergic, dopaminergic, noradrenergic, and cholinergic systems have been implicated in the processes that mediate reactivity to unfamiliar environments (Aloisi et al., 1997; Ceccarelli et al., 1999; Dalley \& Stanford, 1995; Feenstra et al., 1995; Gruen et al., 1996; Hooks \& Kalivas, 1995; Rebec et al., 1997). Of main interest in the present report is the role of the cholinergic system in environmental familiarization. Using in vivo microdialysis, Aloisi et al. (1997) found that acetylcholine (ACh) levels in the hippocampus of rats increased above baseline when the rats were placed into a novel environment. Importantly, as various behavioral measures like rearing and line crossing decreased (i.e., indices of familiarization) so did hippocampal ACh levels. This result suggests a role for $\mathrm{ACh}$ and the hippocampus in learning processes required for environmental familiarization (see also Ceccarelli et al., 1999, and Thiel et al., 1998). Consistent with this suggestion is the substantial literature showing the importance of acetylcholine and the hippocampus in learning and memory (e.g., Kim \& Levin, 1996; Levin \& Simon, 1998; McNaughton \& Morris, 1987; Ohno et al., 1993).

In the present series of experiments we had several goals. One goal was to determine the feasibility of using a measure of environmental familiarization that took advantage of a rat's tendency to differentially interact with a novel object depending on the degree of familiarity with the environment (Experiments 1 and 2). The procedures were based, in part, on an experiment conducted by Sheldon (1969). In that experiment she used a Y-maze apparatus that allowed rats to choose between an arm that contained a familiar object and an arm that contained a novel object. When the environment was unfamiliar, rats went to the arm containing the familiar object. However, as 
the Y maze became more familiar on subsequent trials, rats increasingly chose the arm containing the novel object. We simplified the procedure by only introducing a novel object into an environment after rats were allowed to briefly explore the environment. Presumably, rats less familiar with the environment should interact less with the introduced object. A second goal was to determine whether acute treatment with the nicotinic ACh receptor agonist nicotine would alter environmental familiarization and to determine whether this effect was dose dependent (Experiment 1). Depending on the nature of the task, researchers have found enhancement, retardation, or no effect of nicotine on learning and memory (Dunnett \& Martel, 1990; Levin et al., 1997; see Levin \& Simon, 1998, for a review). Finally, we compared the alteration of environmental familiarization induced by acute nicotine treatment to that of chronic nicotine treatment (Experiment 2). There is some evidence indicating that the neural substrates that mediate the initial effects of nicotine differ, in part, from the processes that mediate the chronic effects of nicotine (e.g., Nisell et al., 1997; Stolerman et al., 1995).

\section{EXPERIMENT 1 Method}

\section{Animals}

The animals were 44 naive male Sprague-Dawley rats (mean, $360 \mathrm{~g}$ ) from a breeding colony at the University of Nebraska. They were housed separately in plastic tubs lined with wood chip bedding in a colony on a 12-h light:dark cycle. Experiments were conducted during the light portion of the cycle. Rats had free access to food and water in the home cages and were handled at least 1 min per day for 3 days before the start of the experiment.

\section{Drugs}

(- )-Nicotine-di-D-tartrate (Research Biochemicals Int., Natick, MA) was mixed in saline $(0.9 \%$ $\mathrm{NaCl}$ ) and then brought to a $\mathrm{pH}$ of $7.0 \pm 0.2$ with a dilute sodium hydroxide solution. Injections were subcutaneous ( $\mathrm{sc}$ ) at a volume of $1 \mathrm{~m} 1 \mathrm{Jkg}$. Nicotine doses were based on the salt form of the drug.

\section{Apparatus}

The environment was an elevated stainless steel platform $88.5 \mathrm{~cm}$ long and $30 \mathrm{~cm}$ wide with a 4.4- $\mathrm{cm}$ lip around its entire edge. The platform was $125 \mathrm{~cm}$ off the floor and was located approximately in the center of a room separate from the colony. Fluorescent ceiling lights provided general illumination and a white noise generator masked external sounds. The novel object was a peach-shaded paint roller ( $7.5 \mathrm{~cm}$ long, $4 \mathrm{~cm}$ in diameter) attached to a green plastic scouring pad ( $9 \mathrm{~cm}$ diameter). All sessions were videotaped for later observations (see Behavioral Measures). Three lines were marked on the platform floor to aid in conducting the experiment and the later observations. One line bisected the platform into equal halves (i.e., midline). The two remaining lines were drawn $10.5 \mathrm{~cm}$ from each end of the platform (i.e., placement areas). These lines were used to guide placement of the rat and the novel object. 


\section{Procedure}

On Days 1 and 2, rats received a sc saline injection or one of three doses of nicotine $(0.2,0.6$, or $1.8 \mathrm{mg} / \mathrm{kg}) 10 \mathrm{~min}$ before each daily placem nt on the elevated platform. After $2 \mathrm{~min}$ on the platform, each rat was returned to the home cage. A fifth set of rats (Group Unfamiliar) was not exposed to the platform on Days 1 and 2. Rather, this group received a daily injection of saline in the home cage. On the test day (Day 3), all rats received a sc injection of saline $10 \mathrm{~min}$ before placement on the platform for $2 \mathrm{~min}$. Located on the opposite end of the platform (in the center of the placement area) was the novel object. This test was the first time Group Unfamiliar had been on the platform. Thus, Group Unfamiliar provides a benchmark by which to determine whether nicotine treatment on the first 2 days altered environmental familiarization. We implemented several procedures designed to minimize the influence of odors on the platform. The end of the platform on which rats were placed was balanced as much as the sample size allowed ( $n=8-10$ per group). Moreover, at the start of each day a rat not included in the experiment was allowed to explore the elevated platform for at least 2 min. Finally, the tray was wiped clean with a wet sponge and then dried after each rat.

\section{Behavioral Measures}

For the familiarization and test days, an observer naive to the group assignments of the rats scored the time contacting platform edge, number of midline crosses, and number of $90^{\circ}$ turns for each rat. Edge contact was defined as any time at least one paw contacted the raised edge of the platform. A midline cross was scored each time a rat's two front paws crossed the line that bisected the platform. A $90^{\circ}$ turn was scored each time the rat's nose moved $90^{\circ}$ from its placement position. Each rat was placed on the platform with its side against the placement area edge and nose facing either west or east (i.e., parallel to the narrower portion of the platform). Thus, movement that resulted in the nose pointing from the east to the north (parallel to the long portion of the platform) was scored as one turn. If the nose shifted from the east to the west, that movement was scored as two turns. We developed this measure in response to Brudzynski and Krol's (1997) data indicating that turning behavior may serve as a more sensitive measure of familiarization than line crosses. However, King's (1999) operational definition of turning more closely fit our definition. In that report, there was no systematic change in turning when it was employed as a measure of exploration on an elevated plus maze.

Time spent interacting with the novel object was scored on the test day. Object interaction was defined as any "directed" contact with the object. Directed contacts mostly include use of the front paws and/or nose. This definition excludes such accidental contacts as backing into the object or brushing against the object with the side (cf. Besheer et al., 1999; Bevins et al., 1997). A second individual naive to the rats' group assignment assessed reliability of the primary rater. This secondary rater scored each behavior for 43 of the rats during the test. The correlation between the two observers was high for each behavior: crosses $(r=.96)$, turning $(r=.83)$, edge contact $(r=.93)$, and object interaction $(r=.97)$.

\section{Data Analyses}

For each rat, an average of each dependent measure was obtained for the familiarization phase (Days 1 and 2). Kruskal- Wallis tests were used for overall analyses of each dependent measure 
during familiarization and testing. If there was a statistically significant main effect, pairwise Mann-Whitney tests were used to compare the control to each experimental group. Statistical significance was set at a two-tailed $\alpha$ of 0.05 for all tests. Nonparametric statistics were employed because of the small sample size and the violation of heterogeneity of variance assumed by parametric statistics.

\section{Results and Discussion}

Figure 1 A shows the median number of midline crosses for each group during the familiarization phase. There was a significant main effect of group, $H(3)=11.34, p=.01$. Subsequent pairwise comparisons between saline-treated animals and each dose of nicotine revealed significantly fewer midline crosses in rats treated with the $1.8 \mathrm{mg} / \mathrm{kg}$ dose of nicotine, $U=6, p=.0025$. Figure IB shows the number of $90^{\circ}$ turns for each group. Acute nicotine treatment did not alter turning, $H(3)=4.31, P=.2303$. Figure $1 \mathrm{C}$ shows the time spent contacting the platform edge. There was a significant group effect, $H(3)=18.57, p=.0003$. Relative to the saline-treated control group, all doses of nicotine decreased the time rats spent on the outer edge of the environment, Us $\leq 12$, ps $<$ .038 .

Figures 2A-2D show for each group the results of the drug-free test. Groups did not differ statistically on midline crosses, $H(4)=8.93, p=.0629$, number of turns, $H(4)=3.31, p=.5071$, or time contacting platform edge, $H(4)=8.31, p=.081$. In contrast, the overall Kruskal-Wallis test on object interaction time was significant, $H(4)=10.93, p=.0273$. As expected, rats that had not previously experienced the platform (Group Unfamiliar) interacted less with the novel object than saline-treated controls that had experienced the platform on two previous occasions, $U=14, p=$ .036 . Rats previously treated with the low dose of nicotine $(0.2 \mathrm{mg} / \mathrm{kg})$ during environmental preexposure also spent more time interacting with the novel object than Group Unfamiliar, $U=12, p$ $=.0206$. As measured by object interaction, acute treatment with the two higher doses of nicotine interfered with environmental familiarization. These two groups $(0.6$ and $1.8 \mathrm{mg} / \mathrm{kg})$ were not statistically different from Group Unfamiliar, Us $2 \geq 21$.

\section{EXPERIMENT 2}

In Experiment 1 we found that acute treatment with nicotine (initial two exposures) interfered with environmental familiarization. In Experiment 2 we sought to replicate the effects of acute nicotine $(0.6 \mathrm{mg} / \mathrm{kg})$ and to determine whether chronic nicotine had a similar effect on the processes involved in environmental familiarization. Moreover, the control groups in Experiment 1 were not treated with nicotine. Thus, any effect in nicotine-treated rats on the test day may be attributable to nonspecific effects of simply receiving nicotine rather than more specific alteration of familiarization. Another goal of Experiment 2 was to assess this possibility by employing controls (acute and chronic) that were equally exposed to nicotine, but in a manner that kept the exposure to the environment temporally separated from nicotine.

\section{Methods}

\section{Animals and Apparatus}

The animals were 50 naive male Sprague-Dawley rats (mean, $341 \mathrm{~g}$ ) from Harlan Industries (Indianapolis, IN) or the University of Nebraska breeding colony. Housing, care, and apparatus were 

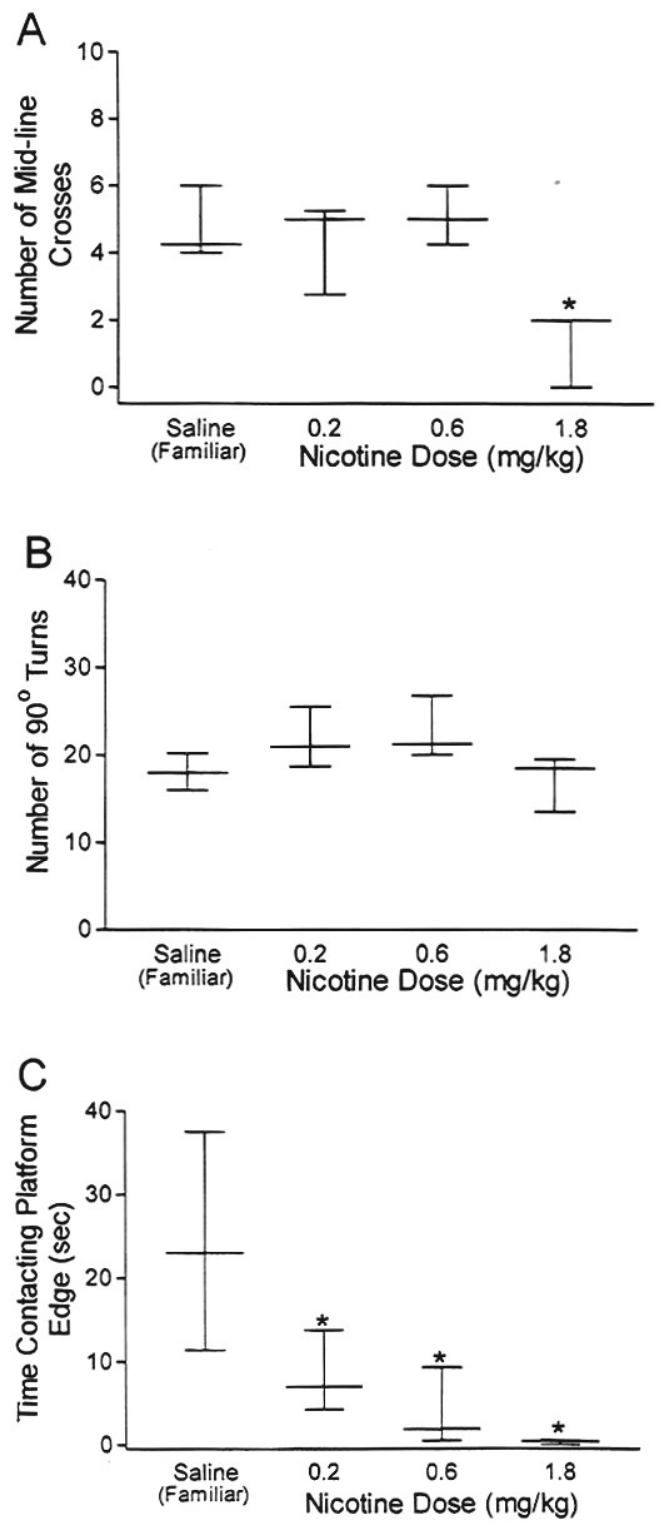

FIG. 1. (A-C) The median number of midline crosses, $90^{\circ}$ turns, and time in seconds contacting platform edge, respectively, for each group during the familiarization phase of Experiment 1 are shown. The longer horizontal line denotes the median and the smaller horizontal lines connected by the vertical line represent the interquartile range. Asterisks denote a statistically significant difference from the saline-treated (Familiar) controls using a Mann-Whitney test $(p<.05)$.

unchanged. (- )-Nicotine tartrate (Sigma Chemical Company, St. Louis, MO) was mixed as previously described.

\section{Procedure}

Pretreatment. Rats were assigned to one of two conditions. For 14 consecutive days rats in the chronic condition $(n=24)$ received a daily sc injection of $0.6 \mathrm{mg} / \mathrm{kg}$ nicotine in the colony. Rats 

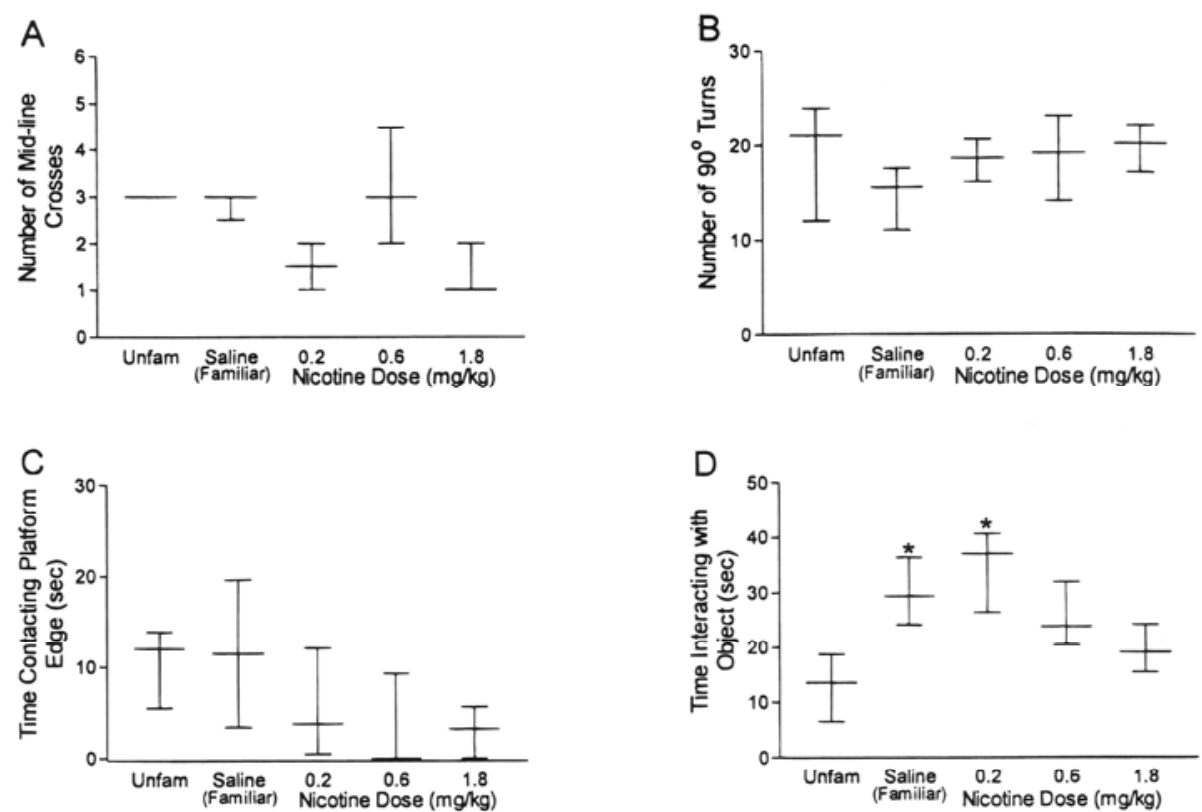

FIG. 2. (A-D) The median number of midline crosses, $90^{\circ}$ turns, time in seconds contacting platform edge, and time in seconds in directed contact with the novel object, respectively, for each group during the drug-free test of Experiment 1 are shown. The longer horizontal line denotes the median and the smaller horizontal lines connected by the vertical line represent the interquartile range. Asterisks denote a statistically significant difference from the Unfamiliar (Unfam) control group using a Mann-Whitney test $(p<.05)$.

assigned to the acute condition $(n=26)$ received a daily sc injection of saline for 14 days.

Familiarization. As in Experiment 1 there were two familiarization days. Rats in the acute condition were divided into three groups. One group $(n=13)$ received a sc injection of nicotine $(0.6$ $\mathrm{mg} / \mathrm{kg}) 10 \mathrm{~min}$ before placement on the platform for $2 \mathrm{~min}$. A second group $(n=7)$ received a sc saline injection 10 min before platform exposure (cf. Group Familiar of Experiment 1). The third group $(n=6)$ also received a saline injection but was never placed on the platform (cf. Group Unfamiliar of Experiment 1). Rats in the chronic condition were also divided into three similarly treated groups, with sample sizes of 11,6, and 7 (i.e., Groups Chronic, Familiar, and Unfamiliar, respectively). Approximately $2 \mathrm{~h}$ after placement on the platform, rats treated with nicotine before platform placement received a saline injection. The remaining rats received a $0.6 \mathrm{mg} / \mathrm{kg}$ injection of nicotine. This procedural detail controlled for exposure to nicotine before the drug-free test.

Testing. The test for environmental familiarization was conducted $24 \mathrm{~h}$ after the end of the familiarization phase. All rats received an injection of saline $10 \mathrm{~min}$ before placement on the platform for $2 \mathrm{~min}$. The novel object was located on the opposite end of the platform.

\section{Results and Discussion}

\section{Familiarization}

We first assessed whether controls in the acute and chronic conditions that were exposed to the platform after a saline injection differed statistically on any of the dependent measures. Familiar- 
ized controls did not differ significantly on any measure, $p s \geq .4452$. Thus, for subsequent analyses and graphing we pooled these rats into one control group (Group Familiar). Figure 3A shows the median number of midline crosses for each group. The overall Kruskal-Wallis was significant, $H(2)=8.49, p=.0143$. According to posthoc Mann-Whitney tests, however, Group Familiar was not statistically different from Groups Acute and Chronic. Figure 3B shows the median number of $90^{\circ}$ turns for each group. Groups did not differ in the number turns, $H<1$. Figure $3 \mathrm{C}$ shows the
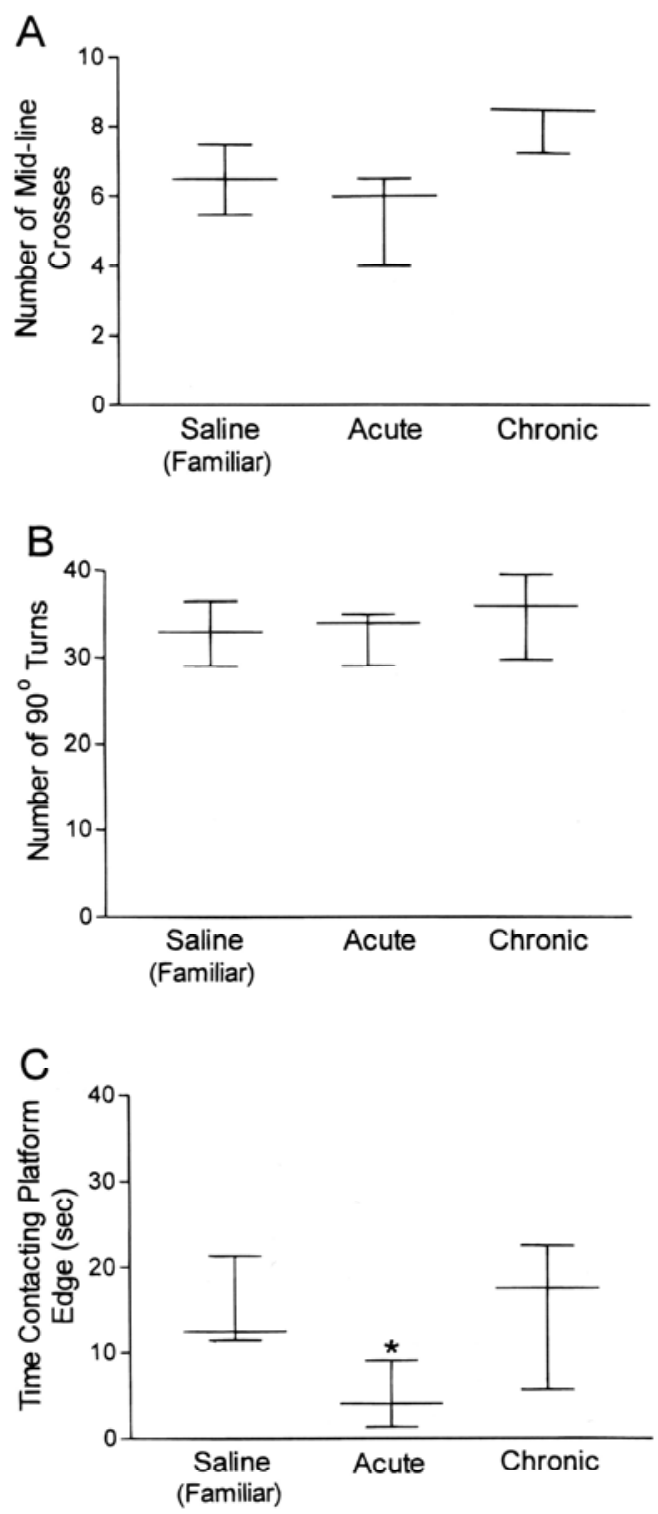

FIG. 3. (A-C) The median number of midline crosses, $90^{\circ}$ turns, and time in seconds contacting platform edge, respectively, for each group during the familiarization phase of Experiment 2 are shown. The longer horizontal line denotes the median and the smaller horizontal lines connected by the vertical line represent the interquartile range. Asterisk denotes a statistically significant difference from the saline-treated (Familiar) controls using a Mann-Whitney test $(p<.05)$. 
median seconds spent contacting the platform edge for each group. There was a significant effect of group, $H(2)=8.92, P=.0116$. Pairwise contrasts revealed that Group Acute spent less time on the platform edge than Group Familiar, $U=31, P=.0066$. Groups Familiar and Chronic were not statistically different.

\section{Testing}

As in the familiarization phase, control rats that were familiarized with the platform (acute and chronic) did not differ on any measure, $p s \geq .9452$. These two groups were pooled for subsequent analyses and graphing (Group Familiar). We conducted a similar analysis between controls in the acute and chronic conditions that were exposed to the platform for the first time on the test day. The two unfamiliarized control groups did not differ significantly from each other on any measure, $p s \geq .2949$, indicating that receiving 16 previous exposures to nicotine did not change the behaviors on the platform relative to a group only receiving nicotine twice. Thus, we also pooled these two controls for subsequent analyses and graphing (i.e., Group Unfamiliar).

Figure 4A shows the median number of midline crosses for each group. The overall KruskalWallis was significant, $H(3)=9.26, p=.026$. Pairwise contrasts revealed that Group Unfamiliar made fewer mid-line crosses than the other three groups, $p s \leq .0307$. The number of turns, $H(3)=$ $6.12, p=.1057$, and the time contacting the edge of the platform, $H(3)=4.06, p=.2554$, did not vary significantly between groups (see Figs. 4B and 4C). Figure 4D shows the median seconds
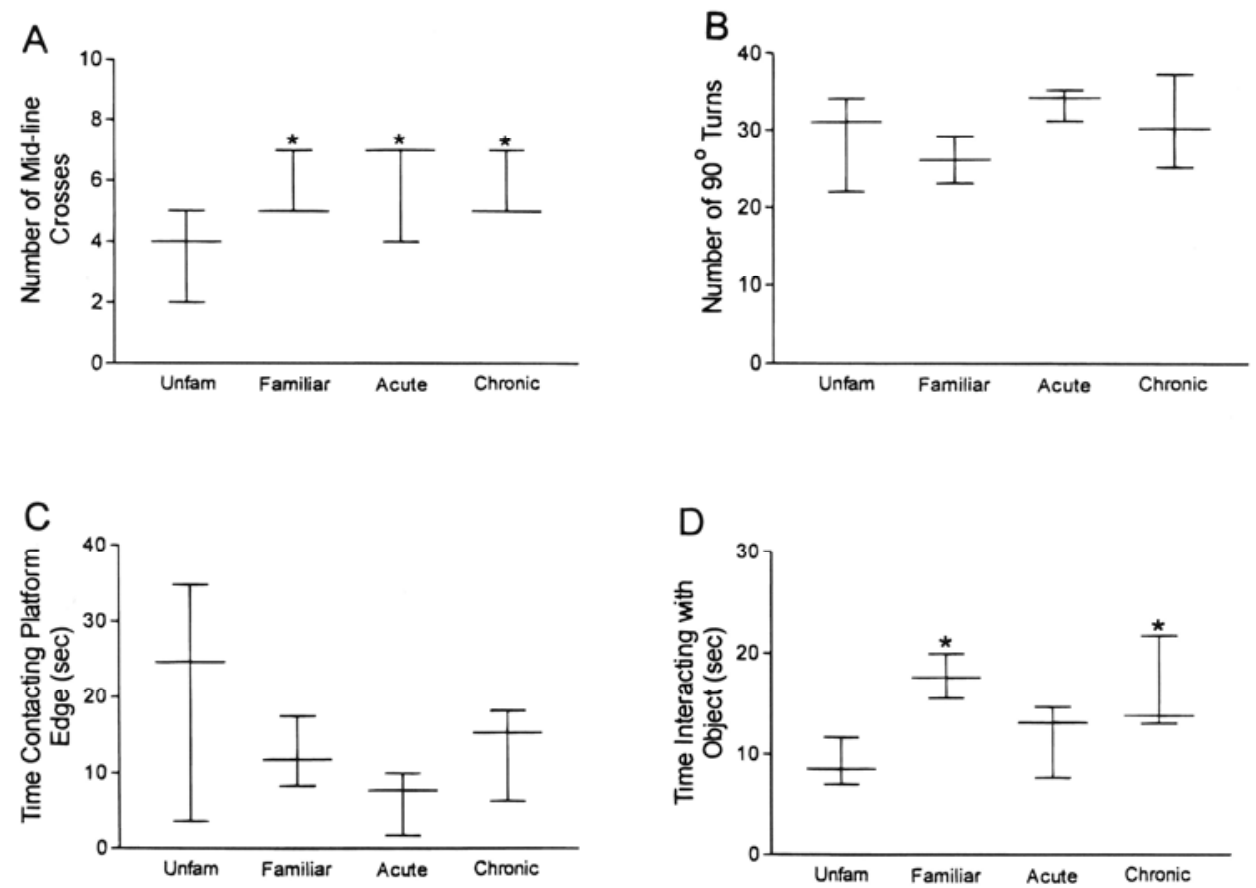

FIG. 4. (A-D) The median number of midline crosses, $90^{\circ}$ turns, time in seconds contacting platform edge, and time in seconds in directed contact with the novel object, respectively, for each group during the drug-free test of Experiment 2 are shown. The longer horizontal line denotes the median and the smaller horizontal lines connected by the vertical line represent the interquartile range. Asterisks denote statistically significant difference from the Unfamiliar (Unfam) control group using a Mann-Whitney test $(p<.05)$. 
spent interacting with the novel object for each group. There was a significant effect of group, $H(3)=10.76, p=.0131$. As in Experiment 1, rats familiar with the platform interacted with the object more than unfamiliar controls experiencing the platform for the first time, $U=23, p=$ .0018. Interestingly, rats treated chronically with nicotine before the familiarization phase also interacted more with the object than unfamiliar controls, $U=35, p=.0371$. In contrast, acute treatment with nicotine interfered with environmental familiarization. Group Unfamiliar and Group Acute were not statistically different, $U=60.5, p=.2282$.

\section{GENERAL DISCUSSION}

In two separate experiments we found that rats that had never experienced the platform (Group Unfamiliar) interacted less with a novel object than rats that had been exposed to an elevated platform for 2 min on two separate occasions without the object (Group Familiar). This data pattern suggests that object interaction can serve as a sensitive measure of environmental familiarization (cf. Sheldon, 1969). Indeed, in Experiment 1 acute treatment during the familiarization phase with the 0.6 or the $1.8 \mathrm{mg} / \mathrm{kg}$ dose of nicotine interfered with processes required for familiarization. Object interaction at these two doses was comparable to that of the Unfamiliar control group. This effect on familiarization was not due to a nonspecific effect of nicotine exposure. A control group in Experiment 2 that received similar exposure to nicotine $(0.6 \mathrm{mg} / \mathrm{kg})$ after platform exposure did not display a decrease in object interaction on the test day like rats in the acute condition.

Nicotine-induced interference with environmental familiarization was not the result of motor impairment during the familiarization phase. First, acute treatment with nicotine, regardless of dose, had no effect on turning. Second, the $0.6 \mathrm{mg} / \mathrm{kg}$ dose interfered with environmental familiarization, yet it had no effect on midline crosses in two separate experiments (cf. $1.8 \mathrm{mg} / \mathrm{kg}$ dose in Experiment 1). The remaining behavioral measure, time spent along the edge of the platform, was decreased by all doses of nicotine. If behaviors that occurred along the edge of the platform were required for familiarization, then rats treated with $0.2 \mathrm{mg} / \mathrm{kg}$ of nicotine should have shown a deficit in environmental familiarization. This did not occur. Rats treated with $0.2 \mathrm{mg} / \mathrm{kg}$ nicotine interacted more with the object than Unfamiliar controls.

Interestingly, there was a dissociation between the acute and chronic effects of nicotine on environmental familiarization. In Experiment 2 we again found that acute treatment with nicotine (0.6 $\mathrm{mg} / \mathrm{kg}$ ) during initial platform placement interfered with familiarization. In contrast, if nicotine $(0.6 \mathrm{mg} / \mathrm{kg})$ was administered daily for 14 days before the environmental familiarization phase (chronic condition), then nicotine treatment 10 min before two separate placements on the elevated platform did not interfere with familiarization. That is, rats in the chronic nicotine condition, like those treated with saline during environmental familiarization, interacted more with the novel object during the drug-free test than controls that had not been exposed to the platform before the test. One interesting possibility is that the present dissociation reflects a difference in statedependent learning (see Overton, 1964). That is, rats in the acute condition were familiar with the elevated platform. However, that familiarity was specific to the interoceptive drug state induced by early treatment with nicotine. Chronic nicotine treatment, in contrast, altered behavioral and/or neural processes such that this state dependency no longer occurred. 
The present research is consistent with a growing literature suggesting that the behavioral and the biological effects of acute and chronic nicotine in part differ. For instance, acute treatment with nicotine can induce a decrease in locomotor activity relative to vehicle controls (Clarke \& Kumar, 1983; Stolerman et al., 1973). Across repeated exposures, however, nicotine has a stimulant effect (Ksir, 1994). Another behavioral dissociation between acute and chronic nicotine was found in Experiment 2. Rats treated acutely with nicotine spent significantly less time than saline controls with at least one paw on the edge of the platform during the familiarization phase. This decrease was not observed for chronically treated rats.

Open and elevated environments (e.g., elevated plus maze) are used in animal models of anxiety (King, 1999; Rodgers, 1997). Perhaps acute and chronic nicotine differentially interact with the anxiogenic/stress effects produced by exposure to the elevated platform. Indeed, acute treatment with nicotine increases plasma levels of corticosterone in rats (Benwell \& Balfour, 1979; Cam \& Bassett, 1984). Summation of the platform-induced stress and the nicotine-induced corticosterone release may interfere with environmental familiarization. Indeed, corticosterone receptors and nicotinic ACh receptors are colocalized in the hippocampus (Morimoto et al., 1996; Takahashi, 1996; Zarei et al., 1999). This brain structure is widely implicated in learning and memory (e.g., Kim \& Levin, 1996; Levin \& Simon, 1998; McNaughton \& Morris, 1987; Ohno et al., 1993). Rats treated chronically with nicotine before initial exposure to the platform would not show this "potentiated stress" effect because nicotine-induced increase in corticosterone shows tolerance after chronic administration (Benwell \& Balfour, 1979; Cam \& Bassett, 1984). Albeit speculative, this potentiated stress effect may also explain the edge avoidance that occurred at all doses of acute nicotine but was not observed following chronic nicotine.

Alternatively, the difference between acute and chronic treatment with nicotine may reflect differential desensitization/inactivation of different nicotinic ACh receptor subtypes or differential increase in the subtype expressed after chronic treatment (Dani \& Heinemann, 1996). For example, chronic nicotine treatment can lead to rapid desensitization and inactivation of nicotinic ACh receptors (Collins \& Marks, 1996; Marks et al., 1993). Also, there is an increase in the number of nicotinic ACh receptors after chronic treatment with nicotine (Marks et al., 1992; Wonnacott, 1990). The mesocorticolimbic dopamine system, via nicotinic ACh receptors located on the ventral tegmental neurons, is also differentially affected by acute and chronic nicotine treatment (Dani \& Heinemann, 1996; Nisell et al., 1994). Using in vivo voltammetry, Nisell et al. (1997) found that the initial intravenous infusion of nicotine increased dopamine levels in the shell and core of the nucleus accumbens. However, rats chronically treated with nicotine showed an increase only in the shell of the nucleus accumbens.

The present report extends previous research on the behavioral effects of nicotine to a new situation. Initial treatment with nicotine during exposure to a discrete environment decreases familiarity with that environment as indexed by object interaction in a drug-free test. Chronic treatment, in contrast, had no effect on environmental familiarization. As described previously, the effects of nicotine on learning and memory are dependent on the nature of the task. Nicotine may enhance, retard, or have no effect on learning and memory (Dunnett \& Martel, 1990; Levin et. al., 1997; Mirza \& Stolerman, 1998; see Levin \& Simon, 1998, for a review). Determining the conditions under which nicotine alters performance may have important implications for the treatment 
of some diseases. Increasingly, nicotine and related compounds have been examined as potential pharmacotherapy for behavioral and cognitive disorders such as Alzheimer's disease, Parkinson's disease, and attention deficit hyperactivity disorder (Conners et al., 1996; Lawrence \& Sahakian, 1998; Newhouse et al., 1997; White \& Levin, 1999).

\section{REFERENCES}

Aloisi, A. M., Casamenti, E, Scali, C, Pepeu, G., \& Carli, G. (1997). Effects of novelty, pain and stress on hippocampal extracellular acetylcholine levels in male rats. Brain Research, 748,219-226.

Benwell, M. E. M., \& Balfour, D. J. K. (1979). Effects of nicotine administration and its withdrawal on plasma corticosterone and brain 5-hydroxyindoles. Psychopharmacology, 63, 7-11.

Besheer, J., Jensen, H. C, \& Bevins, R A. (1999). Dopamine antagonism in a novel-object recognition and a novelobject place conditioning preparation with rats. Behavioural Brain Research, 103, 35-44.

Bevins, R. A., Klebaur, J. E., \& Bardo, M. T. (1997). Individual differences in response to novelty and amphetamine drug discrimination in rats. Behavioural Pharmacology, 8, 113-123.

Bindra, D., \& Spinner, N. (1958). Response to different degrees of novelty: The incidence of various activities. Journal of the Experimental Analysis of Behavior, 1, 341-350.

Brudzynski, S. M., \& Krol, S. (1997). Analysis of locomotor activity in the rat: Parallelism index, a new measure of locomotor exploratory pattern. Physiology \& Behavior, 62, 635-642.

Buccafusco, J. J., Jackson, W. J., Terry, A. v., Marsh, K. C., Decker, M. W., \& Americ, S. P. (1995). Improvement in performance of a delayed matching-to-sample task by monkeys following ABT-418: A novel cholinergic channel activator for memory enhancement. Psychopharmacology, 120, 256-266.

Cam, G. R, \& Bassett, J. R (1984). Effects of prolonged exposure to nicotine and stress on the pituitary adrenocortical response: The possibility of cross-adaptation. Pharmacology Biochemistry and Behavior, 20,221-226.

Ceccarelli, I., Casamenti, E, Massafra, C, Pepeu, G., Scali, C, \& Aloisi, A. M. (1999). Effects of novelty and pain on behavior and hippocampal extracellular ACh levels in male and female rats. Brain Research, 815,169-176.

Clarke, P B. S., \& Kumar, R. (1983). The effects of nicotine on locomotor activity in nontolerant and tolerant rats. British Journal of Pharmacology, 78, 329-337.

Collins, A. C., \& Marks, M. J. (1996). Are nicotinic receptors activated or inhibited following chronic nicotine treatment? Drug Development Research, 38,231-242.

Conners, C K., Levin, E. D., Sparrow, E., Hinton, S. C., Erhardt, D., Meek, W. H., Rose, J. E., \& March, J. (1996). Nicotine and attention in adult attention deficit hyperactivity disorder (ADHD). Psychopharmacology Bulletin, 32, 67-73.

Dalley, J. W., \& Stanford, S. C. (1995). Incremental changes in extracellular noradrenaline availability in the frontal cortex induced by naturalistic environmental stimuli: A microdialysis study in the freely moving rat. Journal of Neurochemistry, 65, 2644-2651.

Dani, J. A., \& Heinemann, S. (1996). Molecular and cellular aspects of nicotine abuse. Neuron, 16, 905-908.

Dunnett, S. B., \& Martel, E L. (1990). Proactive interference effects on short-term memory in rats: 1. Basic parameters and drug effects. Behavioral Neuroscience, 104, 655-665.

Feenstra, M. G. P, Botterbom, M. H. A., \& vanUum, J. E M. (1995). Novelty-induced increase in dopamine release in the rat prefrontal cortex in vivo: Inhibition by diazepam. Neuroscience Letters, 189, 81-84.

Glickman, S. E., \& Hartz, K. E. (1964). Exploratory behavior in several species of rodents. Journal of Comparative and Physiological Psychology, 58, 101-104. Goeders, N. E., \& Guerin, G. E (1996). Effects of surgical and pharmacological adrenalectomy on the initiation and maintenance of intravenous cocaine self-administration in rats. Brain Research, 722, 145-152.

Gould, T. J., \& Steinmetz, J. E. (1996). Changes in rabbit cerebellar, cortical, and interpositus nucleus activity during acquisition, extinction, and backward classical eyelid conditioning. Neurobiology of Learning and Memory, $65,17-34$. 
Gruen, R J., Wenberg, K., Selim, M., Friedhoff, A. J., \& Bradberry, C. W. (1996). Novelty-associated locomotion: Correlation with cortical and sub-cortical GABAA receptor binding. European Journal of Pharmacology, 309, 115-120.

Hooks, M. S., \& Kalivas, P. W. (1995). The role of mesoaccumbens-pallidal circuitry in novelty-induced behavioral activation. Neuroscience, 64, 587-597.

Kim, J., \& Levin, E. (1996). Nicotinic, muscarinic and dopaminergic actions in the ventral hippocampus and the nucleus accumbens: Effects on spatial working memory in rats. Brain Research, 725, 231-240.

Kimble, G. A. (1961). Hilgard and Marquis' conditioning and learning. New York: Appleton-Century-Crofts.

King, S. M. (1999). Escape-related behaviours in an unstable elevated and exposed environment I. A new behavioural model of extreme anxiety. Behavioural Brain Research, 98, 113-126.

Ksir, C. (1994). Acute and chronic nicotine effects on measures of activity in rats: A multivariate analysis. Psychopharmacology, 115, 105-109.

Lawrence, A. D., \& Sahakian, B. J. (1998). The cognitive psychopharmacology of Alzheimer's disease: Focus on cholinergic systems. Neurochemical Research, 23, 787-794.

LeDoux, J. (1998). Fear and the brain: Where have we been, and where are we going? Biological Psychiatry, 44, 1229-1238.

Levin, E. D., Kim, P., \& Meray, R (1996). Chronic nicotine effects on working and reference memory in the 16-arm radial maze: interaction with $\mathrm{D}_{1}$ agonist and antagonist drugs. Psychopharmacology, 127, 25-30.

Levin, E. D., Kaplan, S., \& Boardman, A. (1997). Acute nicotine interactions with nicotinic and muscarinic antagonists: Working and reference memory effects in the 16-arm radial maze. Behavioural Pharmacology, 8, 236242.

Levin, E. D., \& Simon, B. B. (1998). Nicotinic acetylcholine involvement in cognitive function in animals. Psychopharmacology, 138, 217-230.

Marks, M. J., Grady, S. R., \& Collins, A. C. (1993). Downregulation of nicotinic receptor function after chronic nicotine infusion. Journal of Pharmacology and Experimental Therapeutics, 266, 1268-1276.

Marks, M. J., Pauly, J. R, Gross, S. D., Deneris, E. S., Hermans-Borgmeyer, I., Heinemann, S. F., \& Collins, A. C. (1992). Nicotine binding and nicotinic receptor subunit RNA after chronic nicotine treatment. Journal of Neuroscience, 12, 2765-2784.

Mason, K., Heal, D. J., \& Stanford, S. C. (1998). The anxiogenic agents, yohimbine and FG 7142, disrupt the noradrenergic response to novelty. Pharmacology Biochemistry and Behavior, 60, 321-327.

McNaughton, B. L., \& Morris, R G. M. (1987). Hippocampal synaptic enhancement and information storage within a distributed memory system. Trends in Neuroscience, 10, 408-415.

Mirza, N. R, \& Stolerman, 1. P. (1998). Nicotine enhances sustained attention in the rat under specific task conditions. Psychopharmacology, 138, 266-274.

Montgomery, K. C. (1953). Exploratory behavior as a function of "similarity" of stimulus situations. Journal of Comparative and Physiological Psychology, 46, 129-133.

Morimoto, M., Morita, N., Ozawa, H., Yokoyama, K., \& Kawata, M. (1996). Distribution of glucocorticoid receptor immunoreactivity and mRNA in the rat brain: An immunohistochemical and in situ hybridization study. Neuroscience Research, 26, 235-269.

Newhouse, P. A., Potter, A., \& Levin, E. D. (1997). Nicotinic system involvement in Alzheimer's and Parkinson's diseases: Implications for therapeutics. Clinical Pharmacology, 11, 206-228.

Nisell, M., Marcus, M., Nomikos, G. G., \& Svensson, T. H. (1997). Differential effects of acute and chronic nicotine on dopamine output in the core and shell of the rat nucleus accumbens. Journal of Neural Transmission, 104, 1-10.

Nisell, M., Nomikos, G. C., Svensson, T. H. (1994). Systemic nicotine-induced dopamine release in the rat nucleus accumbens is regulated by nicotinic receptors in the ventral tegmental area. Synapse, 16, 36-44.

Ohno, M., Yamamoto, T., \& Watanabe, S. (1993). Blockade of hippocampal nicotinic receptors impairs working memory but not reference memory in rats. Pharmacology Biochemistry and Behavior, 45, 89-93.

Overton, D. A. (1964). State-dependent or "dissociated" learning produced with pentobarbital. Journal of Comparative and Physiological Psychology, 57, 3-12. 
Rebec, G. V., Christensen, J. R. C., Guerra, C., \& Bardo, M. T. (1997). Regional and temporal differences in real-time dopamine efflux in the nucleus accumbens during free-choice novelty. Brain Research, 776, 61-67.

Rodgers, R. J. (1997). Animal models of 'anxiety': Where next? Behavioural Pharmacology, 8, 477-496.

Sheldon, A. B. (1969). Preference for familiar versus novel stimuli as a function of the familiarity of the environment. Journal of Comparative and Physiological Psychology, 67, 516-521.

Stolerman, I. P., Fink, R., \& Jarvik, M. E. (1973). Acute and chronic tolerance to nicotine measured by activity in rats. Psychopharmacologia, 30, 329-342.

Stolerman, I. P., Garcha, H. S., \& Mirza, N. R. (1995). Dissociations between the locomotor stimulant and depressant effects of nicotinic agonists in rats. Psychopharmacology, 117,430-437.

Takahashi, L. K. (1996). Glucocorticoids and the hippocampus. Molecular Neurobiology, 13, 213-226.

Thiel, C. M., Huston, J. P., \& Schwarting, R. K. W. (1998). Hippocampal acetylcholine and habituation learning. Neuroscience, 85, 1253-1262.

White, H. K., \& Levin, E. D. (1999). Four-week nicotine skin patch treatment effects on cognitive performance in Alzheimer's disease. Psychopharmacology, 143, 158-165.

Wonnacott, S. (1990). The paradox of nicotinic acetylcholine receptor upregulation by nicotine. Trends in Pharmacological Science, 11, 216-219.

Zarei, M. M., Radcliffe, K. A., Chen, D., Patrick, J. w., \& Dani, J. A. (1999). Distributions of nicotinic acetylcholine receptor $\alpha 7$ and $\beta 2$ subunits on cultured hippocampal neurons. Neuroscience, 88, 755-764. 